\title{
The Influence of Language on False Memory
}

\author{
Beiyi Cai ${ }^{1, \dagger}$ Yu Zheng ${ }^{2, \dagger, *}$
}

\author{
${ }^{1}$ School of Continuing Education, SJTIU, Shanghai, China \\ ${ }^{2}$ Ecnuas Affiliated Bilingual School, Shanghai, China \\ *Corresponding author. Email: ${ }^{1}$ guanghua.ren@ gecacademy.cn \\ ${ }^{\prime}$ These authors contributed equally.
}

\begin{abstract}
False memory is often happened in life and has attracted lots of attention from researchers. The purpose of this study is to examine the effects of different languages (i.e., mother language and other languages) on false memories. Different word cards were prepared to test which language caused the most serious false memory between 20 female participants. The results showed that the reaction time of the control group (mother language) is shorter, but the accuracy of the mother language is $79 \%$, lower compared to the reaction time of the experimental group (a second language;83\%). And the experiment concludes that the mother language is more likely to produce false memory than the other language. However, there is not enough time for us to do more experiments with other variables, such as balance the gender or ask foreigners to do the test, to ensure internal validity and the reliability of the experiment.
\end{abstract}

Keywords: memory, false memory, language, Chinese participants

\section{INTRODUCTION}

\subsection{Structure of Memory}

Memory, defined in cognitive psychology, is the process of inputting, encoding, storing, and retrieving information. In 1885, memory had been one of the most popular topics that attract many people to investigate. Information processing theory (or information location theory), which emerged in the 1950 s, viewed memory as the process of encoding, storing, and retrieving input information. According to the time of storage, it can be divided into instantaneous memory (Sensory memory 0.24-4second), short-term memory (Working memory 5second-1minute), and long-term memory (More than 1 minute-Forever). We can make short-term memory becoming long-term memory by repeating it over and over again. False memories can occur when mistakes are made in the process of storing memory.

\subsection{False Memory}

In recent years, more and more people have interest in investigating the formation of memory. Kolodner said it's easy to have errors during the memory reconstructive process [1]. False memory happens when people recall or re-recognize events that didn't happen or wrongly recall events that did happen. In 1932, Bartlett discovered false memory for the first time with the experimental methods of serial regeneration and repeated regeneration. From the perspective of cognitive psychology, false memories may be caused by errors in encoding or errors in memory retrieval.

In 1959, Deese first used the quantitative method to study false memory [2]. Until 1995, the work of Rodiger and McDermott [3] led more psychologists to seek a greater understanding of the false memory phenomenon. Since then, there have been a number of experiments on false memories. Such as Loftus and Palmer study, the language used in eyewitness testimony can alter memory [4]. Different studies have also led to different research paradigms in the field of false memory. It can be divided into the centralized associative program paradigm, which tests false memory by semantic association, and the category associative program paradigm tests false memory by categorizing words. This research notes that both of these paradigms are related to language and that the effects of different parts of speech on false memory have been studied before. It turns out that different parts of speech affect false memory. This led us to realize that language could be an important variable affecting false memory. False memory has mostly been studied in monolingual speakers of very diverse languages, eg. Chen et al. in 2008, used Chinese as a kind of language did a false memory experiment[6]. Whether different languages have different effects on false memory is an urgent question to be investigated. It is important to note 
that personal language proficiency depends on their language usage and experience, so it is not a constant feature. Bilingualism and monolingualism would be the two ends of a continuum with no clear division between them due to a lack of consensus on the definition of bilingualism [7] and high variability in its measurement [8]. Therefore, we intend to investigate the false memory of first language and second language through language proficiency[9].

Heredia and Brown [10] investigated how two languages are represented in one brain. Besides, Francis [11] found both languages access a shared conceptual system, and, second, associations between word forms and their concepts are stronger in the first language than in the second language [12].

\subsection{Brief Introduction about This Experiment}

The study investigates the different effects of mother language and second languages on false memories. The hypothesis is that the mother language has less impact on false memories than the second language. The study examined the false memory rates of bilingual Chinese students learning different languages (Chinese as their first language, English as a second language) to see if their first language leads to fewer false memories than their second language. This study uses cards with English and Chinese words and shows cards to subjects. Then different cards are added, and the subjects were asked to identify whether they had seen the words on the cards before. The time between seeing the word for the first time and seeing the word for the second time is 60 seconds.

\section{METHOD}

\subsection{Participants}

There are 20 volunteers (aged between 17 and 30 years old Chinese students) recruited from Jinqiu A-level school of New Channel, including 17 women and 3 men. All twenty students have got 6 on the IELTS test, and they were randomly allocated into two groups. The control group and the experimental group will accept Chinese and English treatment in this experiment to determine the influences of language on false memory. All the participants are bilinguals, and we make sure they know the basic words of Chinese and English.

\subsection{Material and Design}

The experiment we did is the single factor experiment using the between design.

The independent variable is the type of language, which means whether they use their native language (Chinese)or their non-native language (English in this experiment) as their testing language. To be specific, the control group with 10 random participants. They will accept Chinese treatment which all the experiments are conducted in Chinese, their native language. And the experimental group includes other 10 participants who will accept the English treatment during the test which their tests are all conducted in English. The dependent variable in this experiment is the rate of false memory and reaction time.

The materials that we use are five words In each experiment (which are 10 words in total), the Chinese words include Chinese words: 余(being left), 金(gold), 今(today), 戈(shake), 冷(cold)and English words: widow, abroad, expand, beam and steel. While during the test, there are also four similar words to disturb participants' judgment. They are 会 (the last name in Chinese), 全(whole), 令(order), 泠(cool)and English words: window, aboard, bean, and steal.

They are all randomly selected from the dictionary, with no preference for the words, and the standard is that the length of words must be under 8(as easy as possible), and so did we do.

\subsection{Procedure}

The experiment is running on Psychopy (a tool usually used to conduct a psychology experiment). The total experiment takes about 15 minutes. The whole test consists of four parts. (How well they can identify similar words to the true testing words.)

The first part is about preparation for the task. When the experiment starts, each participant is asked to perform this experiment in a room without noises and interruption from others. The participant and the experimenter sit face to face. The participants will be told before the test, "you are going to do a memory test about how well you can remember the words." And they can withdraw from this memory test anytime they want. Then the participant is asked to watch the computer screen. Both experiments for the control group and the experimental group began with an introduction. For the control group, there will be 5 Chinese words together with their meaning written in Chinese (e.g., "泠" means "凉"). For the experimental group, there will be 5 English words together with their Chinese meaning (e.g., "smart" means "㙂明").

The second part is for memorizing the words, and the third part is for resting. After pressing a button to start the test, participants are told to pay attention to the screen and try their best to remember all the words. Each of the words was presented in white font on a neutral black background of psychopy for 5 seconds and disappeared.

After all the 5 words finish revealing, they will have a break time of 60 seconds. The long-time break may cause a vague notion in their memory. And the final part is to test whether they will have a false memory of the words they have remembered in the experiment before. 
After the break, the participants are asked to distinguish whether they have seen the words in the remembering part or not. In case the participants couldn't understand the following steps, we will explain what the participants should do to every participant in Chinese. The words show up randomly (It means they don't show up in the order of 4 seen words and 4, unlike words. E.g., "abroad" is changed into "aboard"). Participants should use the keyboard to tell whether they have seen the word before. They should also do it as fast as possible. The accuracy and speed are all analyzed with excel.

After distinguishing the 8 words, it is the end of the experiment. And then, we would tell him the true aim of the study is to test whether they will form false memory in their brain. We will also ask the participants to have a short interview about how they feel with us.

\section{RESULTS}

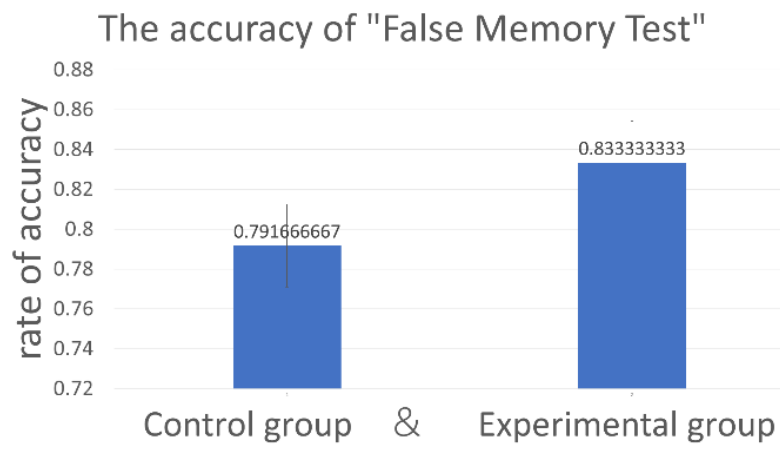

Figure 1. The accuracy of the experiment

The accuracy of the false memory test for the control group is only $79 \%$, and for the experimental group, there has been $83 \%$ of accuracy, according to Figure 1. It means $21 \%$ of the words tested in the control go wrong while only $17 \%$ of all the words in the experimental group go wrong.

\section{Average time used}

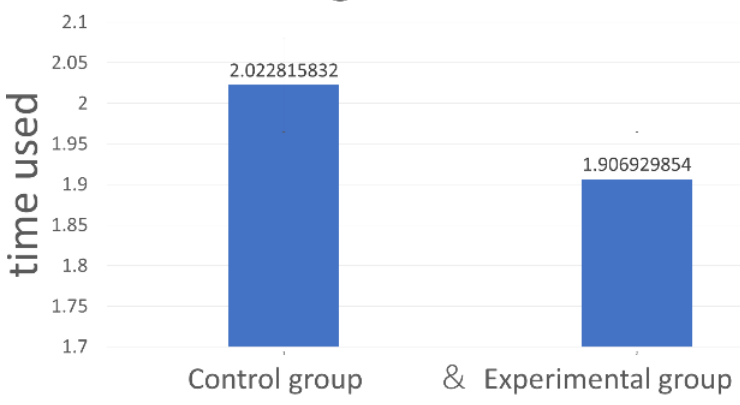

Figure 2. The response time of the experiment

There is a $4 \%$ difference between the two data in Figure 2, representing the time used to do both experiments.

The data references that the difference for average time is little, which is only 0.1 second on average.
So that we can say that time is not a significant influence in the false memory test. $20 \%$ participants will be bothered by the explanation because of the word for test and the similar words.

We can also conclude that people using different means to remember words in our interview are mainly through the shape and sound of the words.

\section{DISCUSSION}

\subsection{Findings of This Experiment}

We did a memory experiment where we asked participants to memorize a few words, and then after a while, we asked them to see several words (Part is recited before, the other part never appears just from the glyph on the very similar words. The results from this experiment fit the hypothesis: participants spend more time thinking when they were memorizing words in other languages, and they made fewer mistakes than their native language.

The experiment found that when recalling words, the participants stayed on the first word for the longest time, and after that, if they become proficient, the stay time would be reduced. In addition, if the participants read out each word when they first read the word, it will also increase their proficiency in the word. Finally, when participants who had lived abroad were asked to memorize these words, it was not difficult for them.

\subsection{Compare to the Previous Study}

According to Daniel's research, they found, the maintenance or "preservation" of false recognition in younger adults under dual task conditions that markedly depressed veridical recognition may indicate that false recognition in younger adults is based on a combination of familiarity and some more specific similarity information [4]. Although Daniel's experiment aimed to investigate older and younger memory, the results of this study are similar to those of Daniel's. False memory can easily be caused by people's stored memories before. Shema's theory can also explain this result. Therefore, we have conducted experiments on false memory in young people, and the reliability of the results is very high. The main reason why people are more likely to have a wrong impression of strange things is simple to understand, which is that they are not yet familiar with new knowledge. Thus, they can only use the stored knowledge to understand, leading to false memories. However, we found from Daniel's experimental results that, as the age increases, the rate of false memory will be higher, 
which may be due to the deterioration of the memory of the elderly.

\subsection{The Strength of This Research}

The strength of the experiment is listed as follows. The most worth mentioning is that this experiment is a laboratory experiment that states that remembering the words was controlled strictly, so the dependent variable only is affected by the independent variable. Then, the experimenter didn't tell participants the real aim of this experiment before the test.Experimenter debriefed after the experiment. This can reduce participants' demand characteristics.

\subsection{The Weakness of This Research and Future Direction}

The first problem is that all participants are Chinese and students, so the result is hard to generalize to other countries' people or other age groups. In order to solve this problem, the experimenter can find more participants with different age groups or from different countries. People can add a foreign experimental group in the future, which means letting other countries people in the experiment group remember words in Chinese and recall them. In the control group, let them remember words in their native language. Then, compare the number of false memories in the end. In this way, the researcher can collect four group participants' data, and the experimental data will be more accurate.

The second problem is this experiment lacks ecological validity. This experiment is a laboratory experiment, so all of the tasks in the experiment are artificial, so it may differ from the problem what one would encounter in real life. In real life, participants will not memorize a few specific words on purpose. In the future, people can add some experimental problems that are related to real life, which will increase the ecological validity of the result.

The third problem is individual differences between each participant. There are individual differences among the experimenters because everyone's memory is different. It is not difficult for people with a good memory to memorize five or ten words at all. The difference in memory will also affect the data. In the future experiment, the researcher can use matched pair method to improve the data, which means different participants are assigned to each condition of the experiment. Still, they are matched on characteristics important to the study. In this way, the researcher can choose people with similar memory to experiment.

\section{CONCLUSION}

In conclusion, this experiment compared the number of false memories in the first language with the number of false memories in other languages and finally found that people have more false memories when using other languages. Therefore, the hypothesis is the same as a result. This research can provide some information to those who are studying language and memory. At the same time, we hope that experimental groups in other languages can be added to improve reliability in future experiments.

\section{REFERENCES}

[1] Kolodner, J.L. (1983). Reconstructive memory: a computer model.Cogn.Sci.7,281-328.

[2] Deese J. Predicting the occurrence of specific speech interference in immediate recall. Experimental Psychology, 1999, 58: 17-22.

[3] Roediger HL, McDermott, KB. Creating false memories: Remembering words not presented in lists. Journal of Experimental Psychology learning, Memory and Cognition,1995,21:803-814

[4] Loftus, E.F., \&Palmer, J.C. (1974). Reconstruction of auto-mobile destruction: An example of the interaction between language and memory. Journal of Verbal Learning and Verbal behavior,13,585589.

[5] JOURNAL OF MEMORY AND LANGUAGE·APRIL $2001 \quad$ Impact

Factor:2.65·DOI:10.1006/jmla.2000.2734

[6] Chen, J.C.W., Li, W., Westerberg, C.E., and Tzeng, O.J.-L. (2008). Test-item sequence affects false memory formation: an event-related potential study. Neurosci. Lett. 431, 51-56.

[7] Edwards, J. (2004). "Foundations of bilingualism," in The Handbook of Bilingualism, eds T.K. Bhatia and W.C. Ritchie (Hoboken, NJ: Blackwell Publishing), 7-31.

[8] Surrain, S., and Luk, G. (2019). Describing bilinguals: a systematic review of labels and descriptions used in the literature between 2005-2015. Bilingual. Lang. Cogn. 22, 401-415.

[9] Suarez M and Beato MS (2021) The Role of Language Proficiency in False Memory: A Mini Review. Front. Psychol. 12:659434.

[10] Heredia, R. R., and Brown, J. M. (2006). " Bilingual memory," in The Handbook of Bilingualism, eds T. K. Bhatia and W. C. Ritchie (Hoboken, NJ: Blackwell Publishing), 225-249.

[11] Francis, W. S. (2020). Shared core meanings and shared associations in bilingual semantic memory: evidence from research on implicit memory. Int. J. Bilingual.24, 464-477.

[12] Gollan, T. H., Montoya, R. I., Cera, C., and Sandoval, T. C. (2008). More use almost always means a smaller frequency effect: aging, bilingualism, and the weaker links hypothesis. J. Mem. Lang. 58, 787814. 